\title{
Prevalencia de depresión en estudiantes de algunas carreras del Centro de Ciencias de la Salud de la Universidad Autónoma de Aguascalientes
}

Terrones Saldivar Ma del Carmen*, Ruiz Esparza Mota José Antonio**, Rosas Cabral Alejandro*, Reyes-Robles Martha Elena*** Prieto Macías Jorge*

\begin{tabular}{|c|c|}
\hline $\begin{array}{l}\text { Resumen } \\
\text { Antecedentes. Los estudiantes del área de la salud, de diferentes } \\
\text { universidades, se enfrentan a situaciones generadoras de ansiedad y } \\
\text { depresión. Objetivo. Con la finalidad de comparar las prevalencias de } \\
\text { depresión entre los estudiantes de las carreras de Médico Cirujano, } \\
\text { Nutrición, Enfermería y Estomatología del Centro de Ciencias de la } \\
\text { Salud de la Universidad Autónoma de Aguascalientes. Metodología. } \\
\text { Se realizó un estudio descriptivo, prospectivo, transversal y compara- } \\
\text { tivo, utilizando el instrumento validado de Zung para evaluar la de- } \\
\text { presión a } 593 \text { estudiantes, a través de un muestreo por conveniencia. } \\
\text { Se realizó estadística descriptiva y para la comparación se empleó la } \\
\text { prueba de X2. Resultados. Encontramos que la prevalencia prome- } \\
\text { dio entre los estudiantes evaluados, fue de I 3.5\%. Observamos un } \\
\text { incremento de la frecuencia de depresión hacia los últimos semestres } \\
\text { en las cuatro licenciaturas evaluadas. Encontramos diferencias signi- } \\
\text { ficativas en las carreras de Nutrición y Estomatología. Conclusión. } \\
\text { Los resultados nos muestran la necesidad de establecer programas } \\
\text { de intervención multidisciplinarios que sean capaces de abordar la } \\
\text { problemática del trastorno depresivo y contribuir a formar mejores } \\
\text { profesionales de la salud. LUX MÉDICA, AÑ09, NÚmERO26, ENERO-ABRIL } \\
\mathbf{2 0 1 4 . P P ~} \mathbf{1 5 - 1 9}\end{array}$ & $\begin{array}{l}\text { Abstract } \\
\text { Background. The students in the area of health, from different univer- } \\
\text { sities, faced with situations that generate anxiety and depression. Ob- } \\
\text { jective. To compare the prevalence of depression between students of } \\
\text { medicine, nutrition, nursing, and dentistry of the University of Aguas- } \\
\text { calientes. Methodology. A prospective, descriptive, transversal and } \\
\text { comparative study was conducted using the validated instrument of } \\
\text { Zung to assess depression to } 593 \text { students, using a sampling by con- } \\
\text { venience, descriptive statistics and X2 test for comparison. Results. } \\
\text { We find that the average prevalence among the evaluated students } \\
\text { was I 3.5\%. We observed an increase in the frequency of depression } \\
\text { towards the last semesters in the four degree courses evaluated. We } \\
\text { found significant differences in nutrition and dentistry students. Con- } \\
\text { clusion. The results show the need for multidisciplinary intervention } \\
\text { programs that are capable of addressing the problem of depressive } \\
\text { disorder and contribute to form the best health professionals. LUX } \\
\text { MÉDICA, AÑ09, NúMER026, ENERO-ABRIL2014.PP 15-19 }\end{array}$ \\
\hline Palab & edicine, Zung. \\
\hline
\end{tabular}

\footnotetext{
Profesor investigador del Departamento de Gineco-Obstetricia y Medicina del Centro de Ciencias de la Salud de la Universidad Autónoma de Aguascalientes.

** Estudiante del noveno semestre de la carrera de Medicina de la Universidad Autónoma de Aguascalientes

*** Técnico de apoyo, del Departamento de Medicina del Centro de Ciencias de la Salud de la Universidad Autónoma de Aguascalientes.

Fecha de recibido: 10 de febrero 2014

Fecha de aceptación: 10 de marzo 2014

Correspondencia: Dra. en C Ma. del Carmen Terrones Saldívar. Profesora investigadora del Departamento de Gíneco-Obstetricia y Pediatría del Centro de Ciencias de la Salud de la Universidad Autónoma de Aguascalientes. Avenida Aguascalientes No 940, Edificio 107 planta alta, Ciudad Universita-
} ria CP 20131, Aguascalientes, Ags., México. Tel +52(449)9108443 Correo electrónico mcterron@correo.uaa.mx 


\section{Introducción}

La depresión es un trastorno afectivo que se caracteriza por un estado de ánimo deprimido, disminución del disfrute, apatía y pérdida de interés en el trabajo, distorsión de los hábitos fundamentales de todo ser humano, generándose insomnio, anorexia, disminución de la libido, de la capacidad de interacción con sus semejantes, irritabilidad, ansiedad y frecuentemente ideación suicida ${ }^{1}$. El trastorno depresivo es una enfermedad que afecta el estado de ánimo y la mente, así como también a la alimentación, al descanso nocturno, a la opinión de sí mismo y a la vida en general. Según cifras de la Organización Mundial de la Salud ${ }^{2}$, en el mundo hay más de 350 millones de personas con depresión, y tiene una distribución cosmopolita. La depresión es una entidad clínica que ocupa uno de los primeros lugares como motivo de consulta en los servicios de psiquiatría. ${ }^{3,4}$ Se calcula que la prevalencia de depresión en México es del $7.4 \%$.

Diversos autores han mostrado preocupación por la salud mental de los estudiantes universitarios, argumentando que el conjunto de las actividades académicas puede influir en su bienestar físico y psicológico. ${ }^{5,6} \mathrm{El}$ ingreso a la universidad constituye un cambio muy importante para los jóvenes, tanto en términos sociales como académicos. El sistema universitario demanda de ellos mayor autonomía, adecuada distribución del tiempo personal, seguridad en la toma de decisiones y adaptación a la nueva cultura organizacional, lo cual afecta su estilo de vida. ${ }^{7}$

Las carreras universitarias del área de la salud han sido reportadas como una de las áreas donde los estudiantes manifiestan mayores niveles de estrés. Los estudiantes de medicina y de otras áreas de la salud, en comparación con otras áreas académicas, están expuestos a estrés social, familiar y académico, entre otros, y son considerados una población vulnerable de padecer ansiedad y depresión ${ }^{5}$. Algunos autores latinoamericanos han publicado que la frecuencia de los cuadros depresivos es mayor en la población estudiantil que en la general, con predominio del sexo femenino, ${ }^{8-10}$ y otros más han reportado que antes de entrar a la escuela de medicina el estatus emocional de los estudiantes se parece al de la población general.,11 El objetivo de este trabajo fue conocer y comparar la prevalencia de depresión en una muestra de estudiantes de las licenciaturas de Medicina, Enfermería, Nutrición y Estomatología.

\section{| | | | | | | | | | | | | | | | | | | | | | | | | | | | | | | | | | | | | | | | | | | | | | | | | | | | | | | | | | | | | | | | | | | | | | | | | | | | | | | | | | | | | | | | | | | | | | | | | | | | | |}

\section{Material y métodos}

Se realizó un estudio descriptivo, transversal, prospectivo y comparativo. Se incluyeron, mediante un muestreo por conveniencia, a los estudiantes del primer y último año de las carreras de medicina $(n=153)$, enfermería $(n=203)$, nutrición $(n=91)$ y estomatología $(n=146)$, durante septiembre y noviembre de 2012. Se utilizó la escala de Zung para evaluar la depresión. La participación de los encuestados fue anónima y bajo consentimiento informado. El 
cuestionario fue aplicado en el salón de clase. El ambiente para contestar la escala fue de tranquilidad, con un periodo de tiempo libre para su llenado, tratando de eliminar cualquier conducta de presión que pudiese interferir con las respuestas de los alumnos, y se respetó la privacidad de sus respuestas. El instrumento de depresión de Zung evalúa algunos de los síntomas de la depresión. El cuestionario consta de 20 afirmaciones, a las que se les da un puntaje que va de 1 a 4 dependiendo de la respuesta: 1 para la respuesta nunca o muy pocas veces; 2 para algunas veces; 3 para frecuentemente; y 4 para siempre. En las preguntas $2,5,6,11,12,14,16,17,18$, y 20 se invierte el orden del puntaje. El puntaje para evaluar la severidad de los síntomas fueron los siguientes: hasta 49 puntos, sin síntomas; más de 50 puntos, depresión leve ó mínima; más de 60 puntos, depresión moderada y más de 70 puntos, depresión severa. Los resultados se expresan en porcentajes y para determinar si hay diferencias en la proporción de manifestaciones depresivas entre las carreras, se utilizó la prueba de $\times 2$.

\section{|| || || ||||||||||||||||||||||||||||||||||||||||||||||||||||||||||||||||||||||||||||||||||||||||||||||||||||||||||||}

\section{Resultados}

Quedaron incluídos 593 estudiantes univerra de medicina, 203 de enfermería, 91 de sitarios del área de la salud, 153 de la carrenutrición y 146 de estomatología (tabla 1).

\section{Tabla I}

Distribución de los estudiantes evaluados, de acuerdo a la carrera que pertenecen

\begin{tabular}{|lccc|} 
& 1er año & Último año & n \\
\hline Medicina & 97 & 56 & 153 \\
\hline Enfermería & 135 & 68 & 203 \\
\hline Nutrición & 59 & 32 & 91 \\
\hline Estomatología & 97 & 49 & 146 \\
\hline & & total & 593 \\
\hline
\end{tabular}

Correspondieron al sexo femenino 368, siendo el $62.0 \%$ y a 225 del sexo masculino, siendo el $37.9 \%$. El promedio de la prevalencia de depresión en el total de estudiantes evaluados fue de $13.5 \%$. Desglosando las frecuencias de depresión del primero y último año de cada carrera, encontramos que para medicina fue de 12.3 y $16 \%$; para enfermería fue de 12.5 y $19.1 \%$, para nutrición, $5 \%$ y $40.6 \%$, y para estomatología fue de $4.12 \%$ y de $16.3 \%$ respectivamente. (figura 1 )

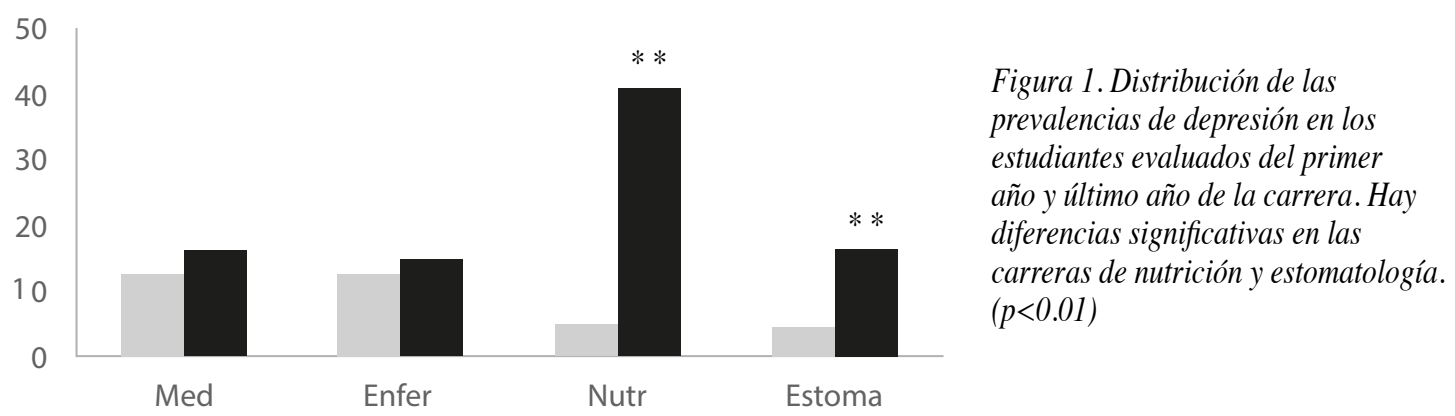


Como podemos observar, en todas las carreras hay una tendencia al incremento de la prevalencia de depresión en los últimos semestres en comparación con el primer año, siendo significativas en los estudiantes de la carrera de nutrición y estomatología $(p<0.01)$.

La depresión predominó en el sexo femenino, en las cuatro carreras. (Tabla 2). Podemos observar que en general, la de- presión leve predominó en todos los casos $(87 \%)$, y el resto fue catalogado como depresión moderada. No encontramos ningún caso de depresión severa. Encontramos además que en la carrera de medicina la depresión es 4.25 veces más frecuente en la mujer que en el hombre; para enfermería la relación fue de 4; en nutrición fue de 15 y para estomatología de 3 .

\section{Tabla 2}

Distribución de la depresión leve y moderada, de acuerdo al sexo

\begin{tabular}{|c|c|c|c|c|c|c|c|c|c|c|}
\hline & \multicolumn{3}{|c|}{$\begin{array}{c}\text { Medicina } \\
(n=153)\end{array}$} & \multicolumn{2}{|c|}{$\begin{array}{c}\text { Enfermería } \\
(n=203)\end{array}$} & \multicolumn{2}{|c|}{$\begin{array}{c}\text { Nutrición } \\
(n=91)\end{array}$} & \multicolumn{2}{|c|}{$\begin{array}{c}\text { Estomatología } \\
(n=146)\end{array}$} & \multirow[b]{2}{*}{ total } \\
\hline & Normal & $\begin{array}{l}\text { Depresión } \\
\text { leve }\end{array}$ & $\begin{array}{l}\text { Depresión } \\
\text { moderada }\end{array}$ & $\begin{array}{l}\text { Depresión } \\
\text { leve }\end{array}$ & $\begin{array}{l}\text { Depresión } \\
\text { moderada }\end{array}$ & $\begin{array}{l}\text { Depresión } \\
\text { leve }\end{array}$ & $\begin{array}{l}\text { Depresión } \\
\text { moderada }\end{array}$ & $\begin{array}{l}\text { Depresión } \\
\text { leve }\end{array}$ & $\begin{array}{l}\text { Depresión } \\
\text { moderada }\end{array}$ & \\
\hline fem & 307 & 12 & 5 & 21 & 3 & 13 & 2 & 9 & - & 65 \\
\hline masc & 187 & 4 & - & 6 & - & 1 & - & 3 & - & 14 \\
\hline
\end{tabular}

\section{Discusión}

Los resultados obtenidos nos demuestran que el trastorno depresivo se va incrementando conforme el estudiante avanza en su carrera. Diferentes estudios realizados por otros investigadores han encontrado que el trastorno depresivo tiende a sufrir una disminución conforme el estudiante está por concluir sus estudios ${ }^{12,13}$. Otro grupo de investigadores también ha encontrado mayor prevalencia de depresión en estudiantes de medicina del primer año cuando los comparan con alumnos del último año de la carrera. ${ }^{14}$ Los alumnos de la carrera de medicina de la UAA son un caso muy diferente, su estado anímico comienza con índices depresivos inferiores a los que muestran alumnos del último año. Los índices para la carrera de enfermería mostraron que la depresión se incrementó en el último año, pero sin ser estadísticamente significativos, los que nos habla de que la depresión se presenta en un número menor de estudiantes. Como se observó, en las carreras de estomatología y nutrición encontramos diferencias significativas en la prevalencia de depresión y ansiedad cuando se compararon los resultados del primer año contra el último año, lo que nos traduce en que hay que considerar que estos problemas son una realidad entre los estudiantes del área de la salud y no exclusivamente en los estudiantes de medicina. Es probable que los estudiantes de estas carreras, de nutrición y de estomatología, que en su mayoría son mujeres, se encuentren en situaciones de estrés más severo, o tengan que cumplir exigencias y expectativas que les solicitan sus carreras. En una universidad privada de Lima, Perú, se encontró en su centro de ciencias de la salud que los estudiantes de medicina tenían los índices de depresión más altos de todas las demás carreras, incluyendo a la de nutrición. ${ }^{12}$ Una de las posibles causas relacionadas con el incremento en los ni- 
veles de depresión y ansiedad en la carrera de nutrición puede ser que en su mayoría son mujeres, y a éstas se les exija a tener óptimas condiciones físicas y mentales por la imagen corporal que les exige su carrera, lo cual las lleva a tener estados depresivos.

Con los resultados de esta investigación se pretende que las autoridades competen- tes tengan la información adecuada para implementar estrategias que conduzcan a diagnosticar oportunamente estos trastornos afectivos y ofrecer programas de apoyo a todos los estudiantes universitarios del área de la salud.

\section{Bibliografía}

1. Villalobos-Galvis, Fredy Hernán. (2009). Validity and reliability of the Positive and Negative Suicidal Ideation Inventory, in colombian students. Universitas Psychologica, 9(2), 509-520.

Disponible en http://pepsic.bvsalud.org/ scielo.php?script $=$ sci_arttext $\&$ pid $=$ S165792672009000200017\&lng=pt\&tlng=en.

2. WHO (OMS) Mental Health and development. Targeting people with mental health conditions as a vulnerable group. WHO Library Cataloguing-inPublication Data. Año 2010

3. Joffre Velázquez, Víctor Manuel, Martínez-Perales Gerardo, Maldonado García Gerardo, Sánchez-Gutiérrez de Lara, Lucero. Depresión en estudiantes de medicina. Resultados de la aplicación del inventario de depresión de Beck en su versión de 13 ítems. Alcmeon, Revista Argentina de Clínica Neuropsiquiátrica, 2007;16(14):86-93.

4. Castilla-Puentes RC, Grau A, Galeno R, Freijo de Mello $M$, Peña N, Sánchez-Russi CA. A multicenter study of major depressive disorder among emergency department patients in Latin-American countries. Depress Anxiwety 2008;25(12):E199-204

5. Arrivillaga, M., Cortés, C, Goicochea, V. \& Lozano, T. Caracterización de la depresión en jóvenes universitarios. Universitas Psicológica, 2004;3:17-25.

6. Feldman, L., Goncalves, L., Chacón Puignau, G., Zaragoza, J., Bagés, N., \& De Paulo, J. (2008). Relaciones entre estrés académico, apoyo social, salud mental y rendimiento académico en estudiantes universitarios venezolanos. Universitas Psychologica, 7(3), 739-752. doi:10.11144/406

7. Flores Ocampo Roberto, Jiménez Escobar Stephany Daniela, Pérez Hernández Sofía, Ramírez Serrano Paula Berenice y Cynthia Zaira Vega Valero. Depresión y Ansiedad en Estudiantes Universitarios. Facultad de Estudios Superiores Campus Iztacala Universidad Nacional Autónoma de México. México, DF. Revista Electrónica de Psicología Iztacala, 2007;10(2):94-105

8. Miranda, Bastidas, Carlos Alberto; Gutierrez, Segura, Julio Cesar; Bernal, Buitrago, Ferney; Escobar, Carlos Andres. Prevalencia de depresión en estudiantes de medicina de la U. del Valle. Revista Colombiana de Psiquiatría,2000; 29(3): 251-260

9. Bohórquez Peñaranda Adriana Patricia. Prevalencia de depresión y de Ansiedad según las escalas de Zung, y evaluación de la asociación con el desempeño Académico en los estudiantes de medicina de la pontificia Universidad javeriana. 2007.Tesis disponible en repository.javeriana.edu.co/bitstream/10554/442/1/med3.pdf?

10. Dávila Figueras Araceli, Ruíz Celis Rocío, Moncada Arroyo Laura, Gallardo Rayo Iris. Niveles de ansiedad, depresión, y percepción de apoyo social en estudiantes de odontología de la universidad de Chile. Revista de Psicología,2011;20(2):147-172

11. Terrones-Saldívar MC, Rosas-Cabral A, GarcíaHuízar MP, Moreno-Castanedo H, Prieto-Macías J, Reyes-Robes ME Comparación de los niveles de ansiedad y depresión entre estudiantes del útlimo año de la carrera de medicina de la UAA e internos de pregrado del estado de Aguascalientes. Lux Médica 2010;5(15):9-14

12. Pereyra-Elías Reneé, Ocampo-Mascaró, Silva-Salazar Javier, Vélez-Segovia Vera, da Costa-Bullón Eduardo, Toro-Polo A. Daniel, Vicuña-Ortega Luis Miguel. Prevalencia y Factores asociados con síntomas depresivos en estudiantes de ciencias de la salud de una universidad privada de Lima, Perú. Revista Peruana de Medicina Experimental y Salud Pública, 2010;27(4):520-526.

13. Al-Busaidi Zakiya, Bhargava Kamlesh, Al-Kindi Rahma, Al-Shafaee Mohammad, Al-Maniri Abdullah. Prevalence of Depressive Symptoms among University Students in Oman. Departamento de medicina familiar y salud pública. Sultan Qaboos, Hospital Universitario Muscat. Sultanate de Oman. Oman Medical Journal 2011;4(26):235-239 DOI 10. 5001/ omj.2011.58.

14. Sidana Surbhi, Kishore Jugal, Ghosh Vidya, Gulati Divyansh, Jiloha RC, Anand Tanu. Prevalence of depression in students of a medical college in New DeIhi: A cross-sectional study. Nueva Dehli, India. Colegio Medico Maulana Azad y Hospital Lok Nayak. Australasian Medical Journal, 2012; 5(5): 247-250. 